\title{
MILLIMETRE MOON MEASUREMENT: 50 YEARS OF LUNAR LASER RANGING SINCE APOLLO 11!
}

\author{
John F. Brock \\ Brock Surveys Pty. Ltd., Parramatta, Australia - brocksurveys@ bigpond.com
}

\begin{abstract}
KEY WORDS: Ancient men of physics, Apollo 11, Lunar Laser Ranging Experiment, Precise Earth-Moon distance, Theory of
\end{abstract} Relativity, International Laser Ranging Service (ILRS).

\begin{abstract}
:
Since the dawn of time the Moon has held fascination for the earliest humans who saw it as a natural navigational beacon, a heavenly body to be revered and a poetic inspiration. Ancient art features the Moon as a prominent subject from all epochs and genres. The name "lunatic" infers that it drives men insane. Giant tides and rapid recessions of water are all attributed to its gravitational influence. As a young boy I was thrilled by stories of Moon travel like Jules Verne's "From the Earth to the Moon" plus TV shows and movies such as "Lost in Space", "Star Trek" and "Dr. Who."

The Russian-American "Space Race" focussed on the exciting possibility of man landing on the Moon. I cannot forget the live telecast of the Apollo 11 astronauts on the Moon's surface in 1969 when I was 13 years old. Four years later I decided to be a land boundary surveyor trained in precise measurement for land title creation. My curiosity was alerted to the Apollo 11 laser ranging aspect of the project when the US team set up a bank of retro-reflectors for measurements from powerful devices on the Earth in the same way we Earthly surveyors make our daily measurements using such EDM equipment.
\end{abstract}

In this paper I will describe the techniques and equipment utilised during this accurate Moon positioning project. You will also see the Earth observatories still measuring to five sites on the Moon and some ancient admirable attempts to determine this distance.

\section{INTRODUCTION}

I am fairly sure that everyone old enough to remember the telecast of the Moon landing by the Apollo 11 crew on $21^{\text {st }}$ July, 1969 will know exactly where they watched the greatest event of the twentieth century. I was thirteen (13) years old when I was transfixed to Neil Armstrong as he descended the ladder from the Lunar Module onto the Moon's surface on the television set in our school library at Liverpool Patrician Brothers College in Australia on that fateful day.

As a most curious star gazer I have always found the wonders of space to be truly amazing so while at University between 197477 studying to be a land surveyor I did Astronomy 1, 2 and 3 which included daylight stars, navigating by the Moon and advanced three dimensional and spherical mathematics. Realising that the bank of lunar laser reflectors placed in the Sea of Tranquility by Buzz Aldrin were for high powered lunar laser rangers to make measurements to the Moon, my interest has always been strong to discover just how accurate these distances have been along with finding out about the types of super powered instruments used for this exercise. The overall

Experiment Package (ALSEP) which included wind and heat monitors, earthquake seismographs and the Lunar Laser Ranging (LLR) Experiment which is the only component of the package still operational due to the fact that the LRRR unit, consisting of 100 reflectors is passive while all of the other elements required batteries which have long since run dead.

\section{UNMANNED SURVEYOR PROBES}

As a preliminary step to plan the landing of men on the Moon, NASA launched a series of unmanned space probes between 1966 and 1968 in what was called the Surveyor Program. Probes named Surveyor 1 to 7 were sent to the Moon as reconnaissance to collect information as well as monitoring the ground and atmospheric conditions in different localities on the lunar surface to ascertain the possibility of a soft surface landing by a manned craft. Surveyor 1 was launched on 30 May and landed on 2 June 1966 at Flamsteed P. Probes 2 and 4 crashed and were lost respectively. Each Surveyor had a television in the equipment while 3 and 7 each carried a soil mechanics surface sampler scoop to dig trenches. Surveyors 5, 6 and 7 had magnets attached to their footpads and an alpha scattering device for chemical analysis of the lunar material. The site chosen by the NASA Planning team to attempt to put the first men on the Moon was the Sea of Tranquility (Mare Tranquillitatus).

As the surface of the Moon which permanently faces the Earth while in its orbit does not change, the aspect that the Moon presents to observers on the Earth always has the Sea of Tranquility fractionally to the upper right of the middle, thus the Laser Ranging Retroreflectors (LRRR) set up by the Apollo 11 crew will continually face the Lunar Laser Rangers positioned at the various sites which come into the frame for shooting the laser light rays which travel there and back in approximately 2.5 seconds covering the $769,000 \mathrm{kms}$ return distance at its average length. 


\section{ANCIENT ASTRONOMERS GET THE LENGTH TO THE MOON}
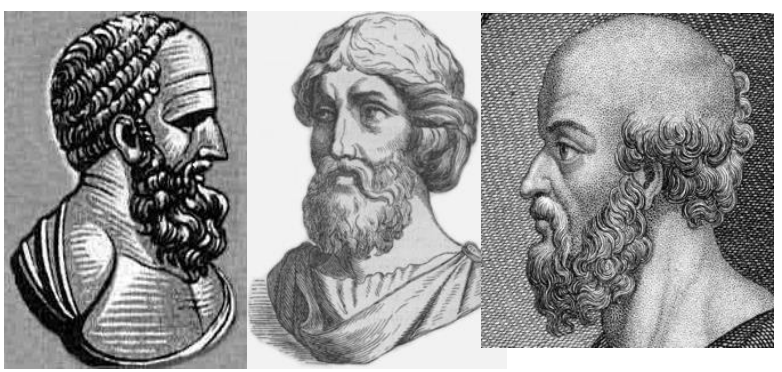

Figure 1. Three Ancient Men of Numbers: Hipparchus,

Aristarchus \& Eratosthenes.

Some ancient astronomers were able to use trigonometrical skill to derive creditable estimates for some of the elements needed to calculate the size of the Earth and Moon as well as their distance apart.

In the third century BC the antiquarian scientist, Aristarchus of Samos (c.310-230 BC), espoused a heliocentric model for our Universe over 1,800 years before the Polish great Nikolaus Copernicus (1473-1543) proposed a similar paradigm in the face of the most improbable Papal doctrines of the flat Earth policy. Another noted mathematician contemporary with Aristarchus was Eratosthenes of Cyrene (c.276-194 BC) whose shadow observations carried out simultaneously at Alexandria and Syene during the summer solstice provided him with the parameters to calculate the Earth's size to a remarkably close figure. Having determined the distance between Alexandria and Syene to be 5000 stadia (abt $800 \mathrm{~km}$ ), that the sun shone directly down a well at Syene (near the Tropic of Cancer) and at the same time a pole at Alexandria cast a shadow showing an angle of $7.2^{\mathrm{O}}$ being $1 / 50$ of a circle, he could decipher that the Earth circumference was 50 x 5000 stadia being 250,000 stadia $(40,000 \mathrm{~km})$. Keeping in mind that the Earth circumference at the Equator is actually $40,075 \mathrm{~km}$ and 40,008 at the poles his result is indeed astounding. (see Figure 2)

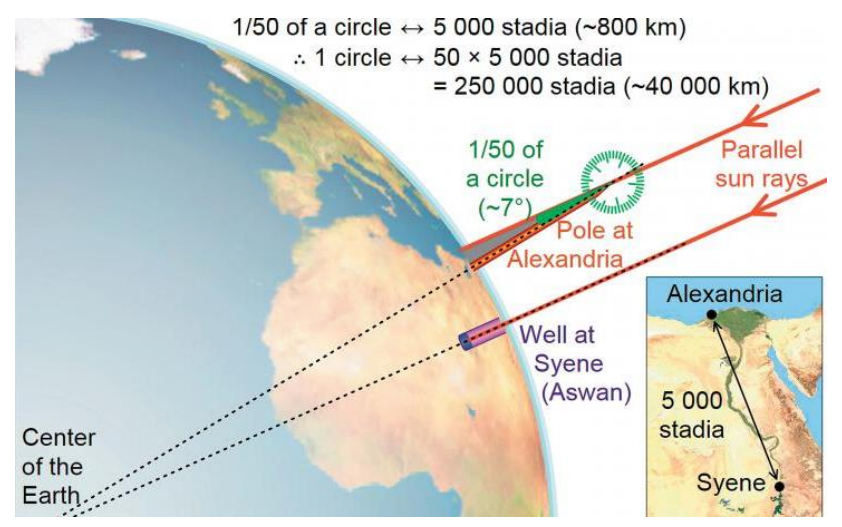

Figure 2. Method of observations devised by Eratosthenes of Cyrene around $220 \mathrm{BC}$ to determine the diameter of the Earth.
Referred to as the Father of Trigonometry, Hipparchus (c.190120 BC) of Nicaea (now in Turkey), advanced the knowledge of the ancients to even higher levels through his now lost treatise "On Sizes and Distances", in which he derives fine results for the Earth-Moon distance, as well as the sizes of each body. Such good linear results are even more surprising when one considers that his planetary model held the Earth at the centre with the Sun and other planets orbiting around it, unlike his predecessor Aristarchus who was an individual well ahead of his time. (Jones A., 2018)

Analysing the more complicated motion of the Moon, Hipparchus constructed a theory of eclipses later used by Ptolemy in his predictive lunar model. Hipparchus had two methods of ascertaining the Moon's position relative to the Earth, the first by observing the difference in parallax between the Moon and the stars rendering an average Earth-Moon distance equal to 77 times Earth's radius (491,106 kms). His second hypothesis used the visually identical sizes of the solar and lunar discs observed during a total eclipse at Syene while $1 / 5$ of the Sun could be seen at Alexandria, yielding a mean Earth-Moon separation equalling 63 times Earth's radius (401,814 kms). (Jones A., 2018) With an actual average closer to 60 by this radius at $382,680 \mathrm{kms}$ (actual current average value is $238,897 \mathrm{mls}-384,467 \mathrm{kms}$ ) the determined amount is most creditable at less than $5 \%$ variance.

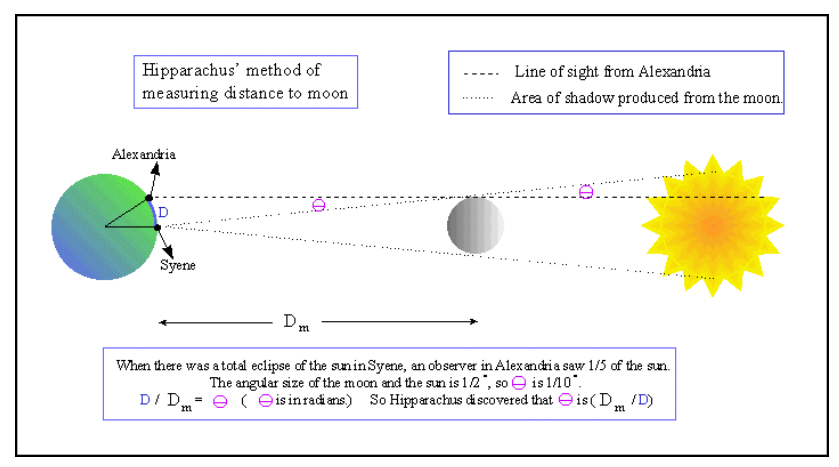

Figure 3. Hipparchus's geometrical solution to estimating the distance from the Earth to the Moon.

\section{INVENTION OF THE GEODIMETER}

Physicist Dr. Erik Bergstrand invented the laser light Geodimeter in 1947 in Sweden, first coming commercially on the market in 1948. Australia's federal mapping agency NatMap purchased one of these first commercial Geodimeters in 1953. When I commenced employment at a private land surveying consultancy in Parramatta in 1973, five separate firms had amalgamated to form Exley, Smith O'Keefe and Partners and we had four AGA Model 700 Geodimeters which was quite unique due to their exorbitant cost. Not only were they very bulky and heavy they were shown to be rather unreliable, especially when the calibration process was not performed fastidiously with some distances logged being up to 0.08 metre in error! In addition to the weight of the 700 we had to carry around unwieldy car batteries as the onboard power supply was only good for a couple of hours! Oh, yes, those were the days! Such bugs in EDM equipment have long since disappeared via the evolutionary cycle in instrument improvement along with reductions in cost and size. 
Seizing upon the potential of this newly created measuring tool, Professor Robert H. Dicke from Princeton University in the USA penned his proposal in a 1962 letter to carry out a series of precise measurements to the Moon bouncing the laser beams from its surface to collect a vast corpus of data to allow scientific analysis of the Earth-Moon relationship to answer some unsolved queries about space.

\section{LUNAR LASER RANGING EXPERIMENT}

2019 was the Fiftieth Anniversary of the start of the Lunar Laser Ranging Experiment by the Apollo 11 team on 21 July, 1969. The total scientific monitoring package was called the Apollo Lunar Surface Experiment Package (ALSEP) consisting of:

1/ ASE Mortar Package Assembly;

2/ Heat Flow Experiment electronic box;

3/ Solar Wind Spectrometer;

4/ Suprathermal Ion Detector/Cold Cathode Ion Gauge;

5/ Lunar Surface Magnetometer;

6/ Charged Particle Lunar Environment;

7/ Passive Seismic Experiment;

8/ Laser Ranging Retroreflector;

9/ Lunar Ejecta and Meteorites Experiment;

10/ Lunar Atmospheric Composition Experiment;

11/ Lunar Surface Gravimeter. (see Figure 4)

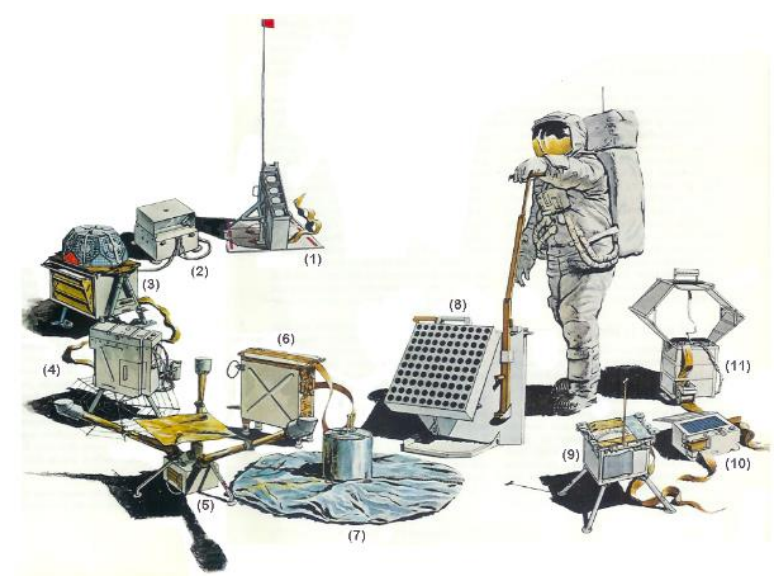

Figure 4. Diagram of the full complement of the ALSEP equipment installed by Buzz Aldrin in 1969.

Experiment 8/ (see list above) has now been continuously operating for over fifty (50) years, being measured by a number of Earth based observatories with three principal bases for the first phase of the project between 1969 and 1985 supplying the data to enable the calculation of the Earth to Moon distance to within about 3 centimetres. It must be pointed out that the orbit of the Moon around the Earth is elliptical with the closest distance at Apogee being around 40,000 kilometres less than its corresponding greatest amount at Perigee. The distance varies from around 360,000 to $400,000 \mathrm{kms}$ and it should also be pointed out that the Moon wobbles in its orbit due to the variable influence of the Earth's gravitational field in a phenomenon known as perturbations. Thus, when referring to a Moon distance as accurate to sub-centimetre level it must be compared to a corresponding measurement taken at that specific instant during its shaky trail around the Earth to a calculated geographic centre of a mathematically constructed spheroidal geometric figure of the lunar body.

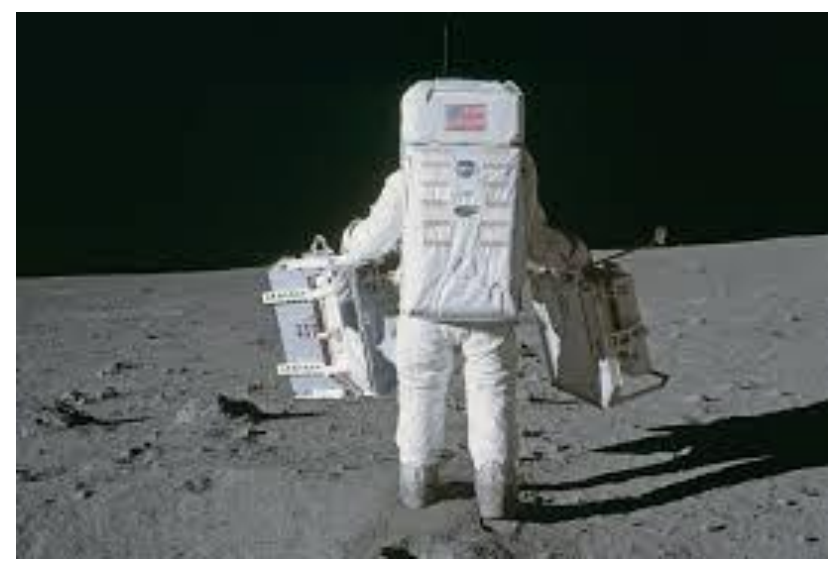

Figure 5. Buzz Aldrin carries the seismograph \& array of Lunar Ranging Retroreflectors (LRRR).

The retroreflector arrays were placed on the Moon in July 1969 by Apollo 11, January and July in 1971 (Apollo 14 and 15) plus French-built units connected to the unmanned rovers by the Soviet Lunakhod program in 1971 and 1973. Design of these retroreflector fixtures for the Apollo units consist of 100 (Apollo $11 \&$ 14) and 300 (Apollo 15) $3.8 \mathrm{~cm}$ diameter corner cubes mounted in an aluminium panel while the Russian type has a lesser number of 14 corner cubes at $11 \mathrm{~cm}$ in size. (Dickey et al, 1994) During the first fifteen (15) years of monitoring, McDonald Observatory in Texas was the only station making routine ranging to the Moon as its $2.7 \mathrm{~m}$ telescope had become active in the late 1960's coming with the commitment to utilize this instrument for a long-term lunar laser ranging program, which has come to fruition as LLR is still performed from this base. (Dickey et al, 1994) The characteristics of the laser light beams originally had an outbound pulse 3.5 metres in diameter and $2 \mathrm{~cm}$ thick with the atmosphere causing divergence of one

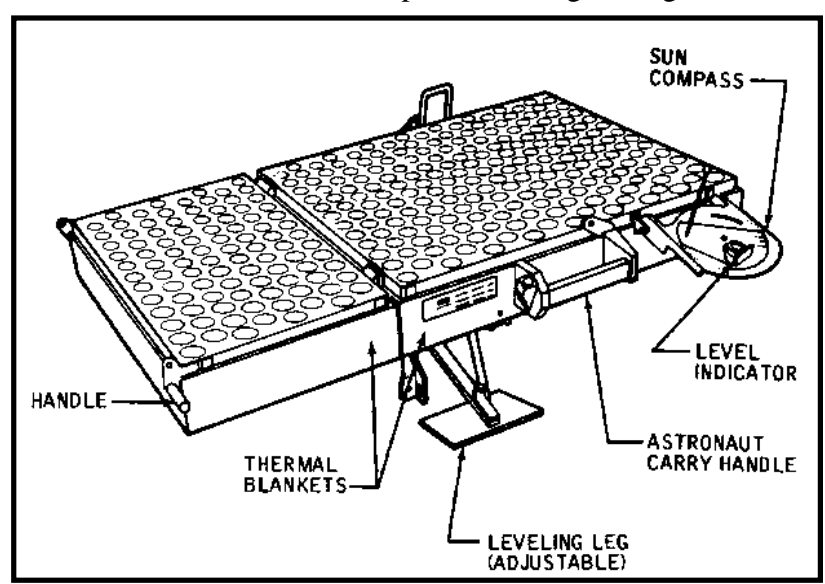

Figure 6. Diagram of Laser Ranging Retroreflector (LRRR) array showing features.

arcsecond, which, at the Moon is $1.8 \mathrm{kms}$. With such a wide dispersal only about 1 in 30 million photons in this range strike the suitcase-sized reflector, with each outgoing laser pulse made up of 300 quadrillion photons. With corner cube diffraction the 
returning beam divergence is about 8 arcseconds causing a footprint about $15 \mathrm{kms}$ across thus allowing only 1 in 30 million of the reflected photons to hit the $3.5 \mathrm{~m}$ mirror. Apollo launched 20 pulses per second for the round trip of 2.5 seconds with about 50 pulses en route during an observing session typically of about 45 minutes duration three times a day. In the early 1970's LLR data achieved an accuracy at the $25 \mathrm{~cm}$ level gradually improving to $15 \mathrm{~cm}$ in the mid 1970's down to 2-3 cms in the middle of the 1980's when the technology moved to the MLRS LLR system. (Uni. Of Texas, 2012)

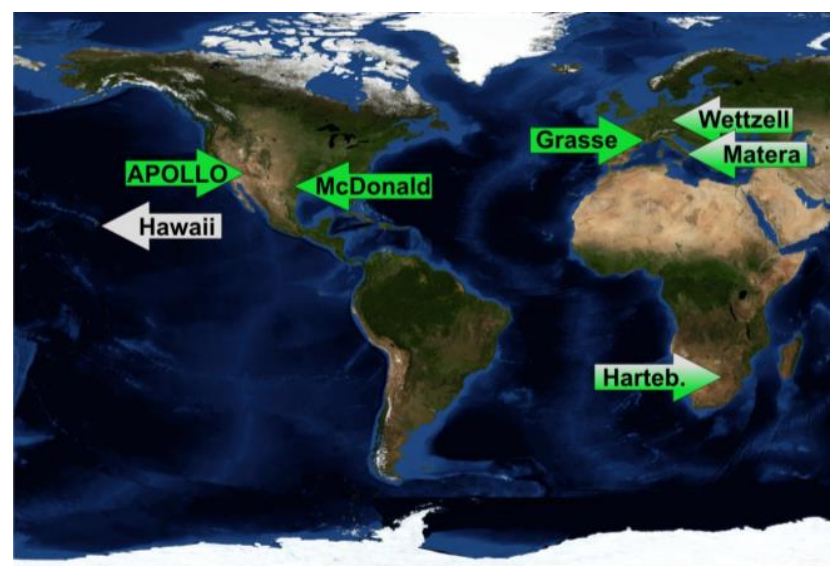

Figure 7. Location of Earth observatories engaged in the Lunar Laser Ranging Experiment.

The three main observing stations on the Earth's surface have been located at Fort Davis (McDonald) in Texas, USA, CERGA (Centre d'Etudes et de Recherches en Geodynamiques et Astronometrie) along the Cote D'Azur in Nice on the French Riviera (shown as Grasse on figure 7) and Mt. Haleakala on the Hawaiian island of Maui. Other observatories such as Orroral (Clark 2011) and Mt. Stromlo in Australia's Capital Territory, South Africa, as well as other US and European installations have the capability to make these long range measurements and have done so in the past. However, only the first three locations have been included in the calculation model of the epoch 196985 for the initial determination of Moon-Earth distances. The sites where lunar ranging retro-reflectors have been placed on the Moon started with the first bank of 100 mounted prisms set by the Apollo $11 \mathrm{crew}$ in the Sea of Tranquility in 1969, as well as four other spots by the unmanned Russian space modules Lunakhods 17 (also 1) and 21 (also 2), together with prism arrays laid down by the Apollo 14 (100) and 15 (300) missions during 1971. Further planning is in hand for the placement of more retroreflectors in selected positions to help improve the geometry of the model from the data as it is received. (see Figure 8).

\section{FIFTY YEARS OF LLR SHOWS A MOVING MOON}

As LLR is the sole survivor from the ALSEP project it is historic from this perspective alone, but the fact it is the first tangible proof of Einstein's Theory of Relativity makes it truly eminent. As Doctor Emmett Brown (played by Christopher Lloyd in the "Back to the Future" trilogy) emphasised the presence of the space-time continuum, Einstein postulated that the Universe was ever-expanding, giving weight to the Big
Bang Theory at the beginning of creation of the Cosmos such that, after the immediate massive explosion billions of years ago, the fragments left floating in the vacuum of space are continuing to drift further apart. This spreading hypothesis has been demonstrated to be real through the precise Earth-Moon measurements down to sub-centimetre accuracy thus establishing that the Moon is moving away from the Earth at a rate of 3.8 centimetres a year. Such a small rate would be classified as imperceptible, as in a hundred years the shift would only be 3.8 metres, so at that rate only over eons of years, like 1 million years, could cause any noticeable effects but even this would only be $3.8 \mathrm{kms}$ over an overall separation of 384,400 $\mathrm{kms}$ which is a mere $0.001 \%$ ! In the surveying profession we are trained and taught that boundaries of real property which adjoin water, like rivers and the sea, are subject to the Doctrine of Accretion and Erosion which instructs us that for the areas of land to suffer loss or addition the process must be natural and imperceptible. Such a definition does not have a basis in absolute numbers, but most definitely $0.001 \%$ is so infinitesimal as to constitute being "imperceptible." Are you aware that there is also displacement of land on the surface of the Earth related to movement in the Tectonic Plates to such a degree that Australia moved 6 centimetres per year for thirty (30) years representing a total shift of 1.8 metres over that period? Although this could also be claimed to be "imperceptible", it has nonetheless caused a change in our national terrestrial coordinate network (AusGeoid 2020), which in turn is to be

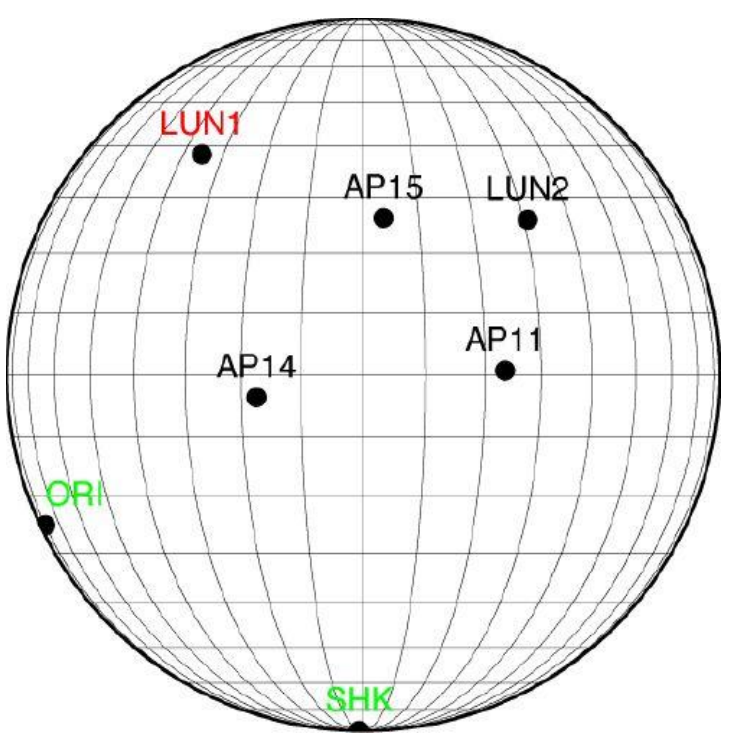

Figure 8. Showing the five lunar sites for LRRR with proposed future positions shown in green.

factored into what is termed the International Terrestrial Reference Frame (ITRF) to keep abreast of the dynamic nature of the Earth's ever-changing relative position with regards to continental drift and its special linkages with its other orbiting neighbours. As Australia moves generally towards the northwest at the rate of 6 centimetres per year the abutting plate is travelling in the opposite direction at a lesser rate, while other crustal segments are shuffling about at differing rates in various routes. Such inconsistent and variable position changes are not sustainable over very long time frames, but history has shown that reversals of movement trends do occur, and as I said 
previously, such minimal changes are not a cause for major concern for many generations to come.

Significant advancements in equipment and techniques came after the first 25 years of LLR activity. In the USA the McDonald Laser Ranging System (MLRS) replaced its older instrument with a Quantel 120 millijoule/pulse 200 picosecond pulse-length Neodymium-YAG laser firing at 10 Hertz. Other features to be adopted are X-Y Offset-Guiding Stage, optical enhancements, Auto-Guiding and Image Enhancement System, Avalanche Photodiode Detector, Disciplined Oscillator and MLRS Control Computer. (Shelus P.J. et al, 1995) With these various enhancements to LLR the level of accuracy is said to be as low as less than a centimetre. The CERGA base is a dedicated LLR facility without the added duty of Satellite Laser Ranging, as is the case at McDonald, so the larger majority of data for Earth-Moon distancing now emanates from the French

\begin{tabular}{|c|c|c|}
\hline Observatory & Period & Obsns \\
\hline OMCD6985.DAT McDonald Tel 2.7n & m 1969-85 & 3604 \\
\hline OMCD8388.DAT McDonald MLRS & 1969-85 & 631 \\
\hline OMCD8815.DAT McDonald MLRS & 1988-2015 & 53670 \\
\hline OGRA8486.DAT Grasse (Rubis) & 1984-86 & 1188 \\
\hline OGRA8705.DAT Grasse (YAG) & $1987-2005$ & 8324 \\
\hline OGRA0918.DAT Grasse (MeO) & 2009-2018 & 5747 \\
\hline OHAL8490.DAT Haleakala & 1984-90 & 770 \\
\hline OMAT0318.DAT Matera & 2003-18 & 224 \\
\hline OAPO0616.DAT Apache Point & $2006-16$ & 2648 \\
\hline $\begin{array}{l}\text { OWET2018.DAT Wettzell } \\
\text { TOTAL }\end{array}$ & 2018 & 22 \\
\hline OMCD6918.DAT All observations & $1969-2018$ & 26828 \\
\hline
\end{tabular}

Table 1. List of files of LLR observations \& periods

locality. 1997 archives from the CERGA LLR team can be accessed for results obtained in measuring sessions from the observatories listed with observations made shown in Table One with data file codes to allow individual inspection. (Barache et al, 2019)

LLR stations listed in Table 1. are McDonald observatory (Texas), operational since 1969, Grasse-Calern observatory (OCA - ex CERGA) ranging since 1984, Haleakala (LURE NASA Lunar Ranging Experiment, Maui, Hawaii) in action from 1984 to 1990, Matera base in Italy since 2003, Apache Point observatory (APOLLO, New Mexico) working from 2006 and Wettzell installation involved since 2018. (Barache et al, 2019) University of Hawaii IfA operates and maintains the TLRS-4 Laser Ranging System at the Haleakala High Altitude Observatory Site (HO) on the island of Maui under a contract with NASA/GSFC (Goddard Space Flight Centre). LLR has been carried out here since 1972 when the LURE Observatory was established and running between 1972-2004.

\section{INTERNATIONAL LASER RANGING SERVICE (ILRS)}

The ITRF was established by the International Earth Rotation and Reference Systems Service (IERS) with the formation of another group known as the International Laser Ranging Service (ILRS) tasked with the responsibility to research and develop the necessary global standards and specifications for laser ranging activities, encouraging international adherence to its conventions. After a quarter of a century of lunar tracking by McDonald base discussions on future lunar laser ranging among the affiliated organisations governing outer space monitoring ratified the formation of the ILRS to provide greater connectivity with lunar ranging augmented with Satellite Laser Ranging (SLR) so that the refinement of the model of interspatial coordinate networks can achieve greater standards of precision. The ILRS Governing Board convened its first meeting on 25 September 1998 with John Degnan as GB Chairman presiding over the many functions of space measurement as entrusted to this high profile designation. Further developments in this area have the potential to provide more certainty for future space missions as well as modifying all Earth based systems dependent upon reliable and continuous satellite data such as GPS, along with a greater understanding of the dynamics of the Universe around us.

\section{BUZZ ALDRIN SAVES THE CREW WITH HIS PEN}

Did anyone know that Neil Armstrong does not appear in any of the colour stills shot on the Moon's surface? In fact, ALL of these images are of Buzz Aldrin with Neil as cameraman, with the only vision of Neil appearing as a reflection in Buzz's helmet visor! Another not so well known fact about the Apollo 11 mission is that this team barely made it home alive from their lunar outpost. When it came time for the Lunar Module to take off Buzz found that the ascent engine arming breaker push/pull switch was broken, probably caused by the large space suit backpacks as they moved around their confined cavity. As any metallic object would cause the electronics to short out completely, a quick thinking Buzz used his felt tip pen to engage the circuit breaker, and that is exactly what happened, allowing the duo who were first to walk on the Moon to make it back to the Command vessel then return home to once again walk on the Earth.

\section{CONCLUSION}

Many monumental events have occurred in World history, but there are only a handful which have taken place in the living memory of those who are alive now, so man landing on the Moon would certainly rate as one of the most remarkable feats of mankind for all time. For me, what makes it even more amazing is that I became a land surveyor after witnessing this spectacle as a schoolboy live on TV, and the technology employed to continuously make measurements to the Moon to less than 10 millimetres was developed for the purposes of long range Earth mensuration and later adapted for the purposes of everyday cadastral surveying along the streets of modern cities and thoroughfares. The ongoing Lunar Laser Ranging Program relabelled as the ILRS will continue to record and store the Earth-Moon continuum with even more improved precision than what is already catalogued for a long period into the future.

\section{REFERENCES}

Barache C., Bouquillon S., Carlucci T. \& Francou G., Lunar Laser Ranging Observations From 1969 to 30 November 2018, Paris Observatory Lunar Analysis Centre (POLAC), Observatoire de Paris (SYRTE) - CNRS UMR 8630 - May 2019.

Clark, Philip, Acquisition! The Story of Orroral Valley Space Tracking Station (Philip Clark, Canberra, 2011). 
Dickey, J.O. et al, Lunar Laser Ranging: A Continuing Legacy of the Apollo Program, Invited Review Article, Science Vol. 265, pp. 482-490, 1994.

Gurtner, Werner and Pearlman, Michael, International Laser Ranging Service (ILRS), Report of the International Association of Geodesy 2007-2009.

Jones, Alexander Raymond, Hipparchus - Greek Astronomer, August 17, 2018.

www.britannica.com/biography/Hipparchus-Greek-astronomer

Shelus, P.J., Ricklefs R.L., Ries J.G., Whipple A.L. and Wiant J.R., Lunar Laser Ranging at McDonald Observatory: Beginning the Second Quarter Century (McDonald Observatory, Uni of Texas 1995).

Williams, James G. et al, Lunar Laser Ranging Test of the Equivalence Principle with the Earth and Moon, 2 January 2009.

Web sites: ilrs.gsfc.nasa.gov - Goddard Space \& Flight Centre and www.iers.org

www.csr.utexas.edu $>$ mlrs $>$ history - History of Laser Ranging and MLRS - The Birth of Laser Ranging. (2012). 